\title{
Design of Dual-band Frequency Selective Surface with Miniaturized Elements
}

\author{
Amir Ebrahimi, Student Member, IEEE, Withawat Withayachumnankul, \\ Said Al-Sarawi, Member, IEEE, and Derek Abbott, Fellow, IEEE \\ School of Electrical and Electronic Engineering, The University of Adelaide, SA 5005 \\ amir.ebrahimi@adelaide.edu.au
}

\begin{abstract}
A new dual-band frequency selective surface (FSS) is introduced in this paper. By having a miniaturized unit cell size, the proposed FSS transmission response shows minimal sensitivity to the angle of the incident electromagnetic wave. The two passband frequencies of the proposed configuration can be controlled independently by tunning the unit cell geometrical parameters appropriately. An equivalent circuit model analysis is presented to provide an insightful synthesis procedure for the filter. Full-wave numerical electromagnetic simulations are used to confirm the analysis and synthesis method presented based on the circuit model.
\end{abstract}

Index Terms-Dual-band, Frequency selective surface, periodic structures, spatial filter.

\section{INTRODUCTION}

Frequency selective surfaces are spatial filters that are widely used in radomes for antennas, microwave absorbers, microwave lenses, reflect array antennas etc. [1]-[6]. A frequency selective surface (FSS) is usually formed by periodic arrays of metallic patterns that are printed on dielectric substrates. The important design specifications in FSSs are the low sensitivity of the transmission response with respect to the oblique angles of the incident electromagnetic wave, the low-order harmonic contents of the response, and the ability to operate in close proximity to the radiation source [1].

In advanced microwave systems, e.g. used in aircraft and shipping, multiband FSSs are needed to cover different operational frequency bands [7]. Different schemes are proposed so far for designing multiband FSSs such as: using fractal metallic arrays [8], [9], perturbation techniques [10] and genetic algorithms [11], [12]. In most of the presented structures, the unit cell size or element spacing is comparable to the resonance wavelength and this makes them sensitive to the incident angle of the electromagnetic wave and causes undesirable low-order harmonics in the frequency response. Recently, new types of dual-band frequency selective surfaces have been introduced in [7], [13], [14] that make use of non-resonant elements in their structures. These recent designs show a lower dependency to oblique angles of the incident wave. However, the element sizes are not small enough (around $\lambda_{0} / 6$ ) for the applications where the FSS should be used in very close proximity to the radiation source.

In this paper, a dual-band FSS is designed with the unit cell size as small as $\lambda_{0} / 10$. The proposed FSS is composed of an inductive wire grid and an array of square-loop cells that are printed on the two sides of a thin dielectric substrate. The new

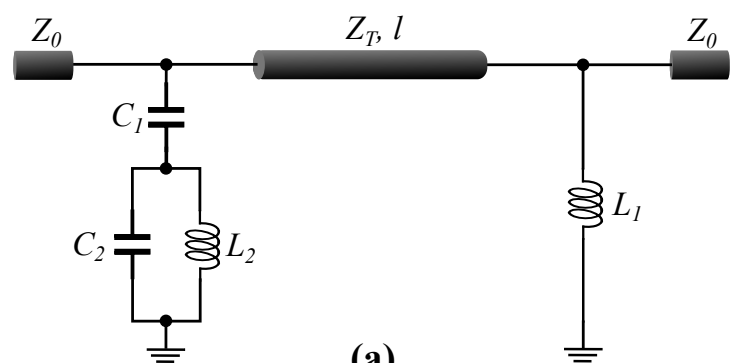

(a)
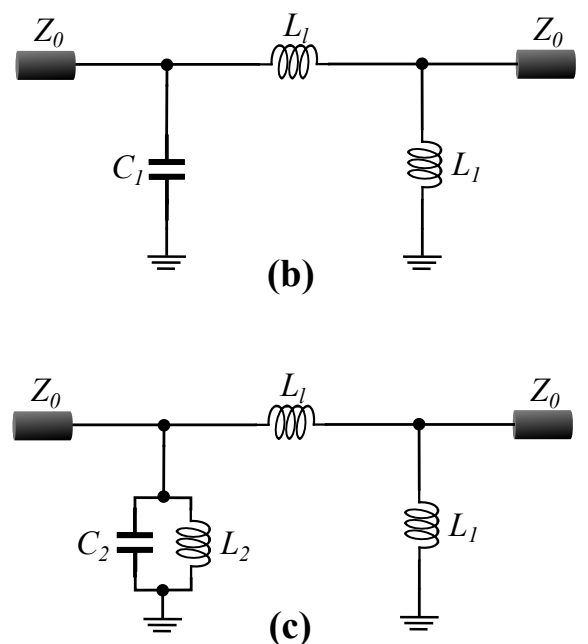

Fig. 1. (a) Equivalent circuit model of the dual-band filter. (b) Simplified circuit model for the first operational passband. (c) Simplified circuit model for the second operational passband.

design provides the advantage of controlling the two passband frequencies independently by varying the physical parameters of the unit cells. The synthesis method and design procedure of the proposed FSS will be presented in the next sections.

\section{The Design PRINCIPLE}

Fig. 1.(a) shows the basic circuit model of the dual-band microwave filters that forms the foundation of the proposed FSS. The filter is composed of a hybrid resonator separated from an inductor through a short piece of transmission line. The hybrid resonator is composed of a parallel $L C$ resonance circuit of $L_{2}, C_{2}$ that is in series with the capacitor $C_{1}$. The transmission line has the length $l$ and the characteristic impedance of $Z_{l}$. The frequency of the first passband is mainly 
determined by $L_{1}, C_{1}$ and the length of the transmission line $l$. On the other hand, the second passband frequency is dominated by $L_{2}, C_{2}$. Fig. 1.(b) shows the equivalent circuit model of the filter for the first passband. In this model, it is assumed that the two passbands frequencies are sufficiently seperated so that, $L_{2} C_{2}$ possesses a low impedance and can be ignored. The transmission line $(l \ll \lambda)$ is replaced with $L_{l}$ inductor. Therefore, by using a simple circuit analysis, the frequency of the first passband can be found as

$$
f_{1} \approx \frac{1}{2 \pi \sqrt{C_{1}\left(L_{1}+L_{l}\right)}} .
$$

If the relative permeability of the transmission line is considered to be $\mu_{r}, L_{l}$ can be calculated as [1]

$$
L_{l}=\mu_{0} \mu_{r} l \text {. }
$$

So,

$$
f_{1} \approx \frac{1}{2 \pi \sqrt{C_{1}\left(L_{1}+\mu_{0} \mu_{r} l\right)}} .
$$

At the second passband, the parallel $L_{2} C_{2}$ is dominant and the filter can be modelled by the circuit of Fig. 1.(c). For this passband, the effect of $C_{1}$ is ignored and the second passband frequency is approximated as

$$
f_{2} \approx \frac{1}{2 \pi} \sqrt{\frac{1}{C_{2}}\left(\frac{1}{L_{2}}+\frac{1}{L_{1}+L_{T}}\right)} .
$$

The circuit shows a transmission zero that is arisen from the hybrid resonator of $C_{1}, L_{2}$ and $C_{2}$. This zero notch separates the two passbands and improves the out of band rejection of the filter. From Fig. 1.(a), this zero frequency can be obtained as

$$
f_{z}=\frac{1}{2 \pi \sqrt{L_{1}\left(C_{1}+C_{2}\right)}} .
$$

From (1) it is found that the first passband frequency $\left(f_{1}\right)$ is independent of $C_{2}$. Also, (4) shows that $f_{2}$ is not dependent on $C_{1}$. This allows to control the two passband frequencies independently. This characteristic will be studied in detail in simulations.

\section{FSS Synthesis BASED ON THE CIRCUIT MOdEL}

A dual-band frequency selective surface can be synthesized by using the filter model described in the previous section. A 3-D view of the synthesized FSS is shown in Fig. 2. The structure is composed of two metallic arrays that are separated from each other by a dielectric substrate with a thickness of $l$. The back layer is composed of a metallic wire grid, which presents the inductor $L_{1}$ of the equivalent circuit. By interacting with the normal incident electromagnetic wave, this wire grid shows an inductive behaviour where, increasing $w$ or decreasing $D$ will decrease the inductance of $L_{1}$. The top layer on the other side comprises a square-loop array that presents the hybrid resonator $\left(C_{1}, L_{2}, C_{2}\right)$ of the equivalent circuit in Fig. 1. In this pattern, each square-loop cell has a side length of $D-g$ and the adjacent cells are separated from each other

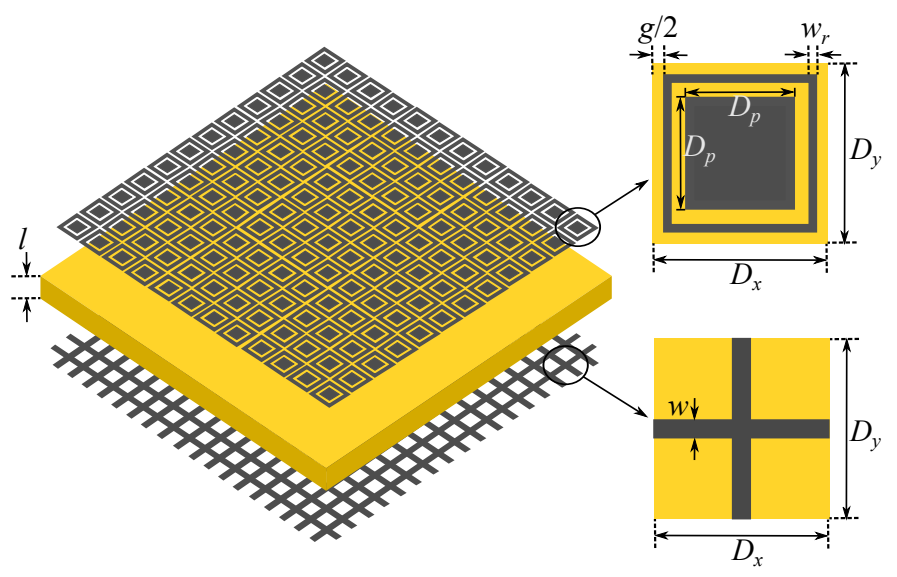

Fig. 2. Topology of the synthesized dual-band FSS. The unit cells of the top and bottom metallic arrays are shown on the right hand side of the figure. The black shading denotes denots the metal.

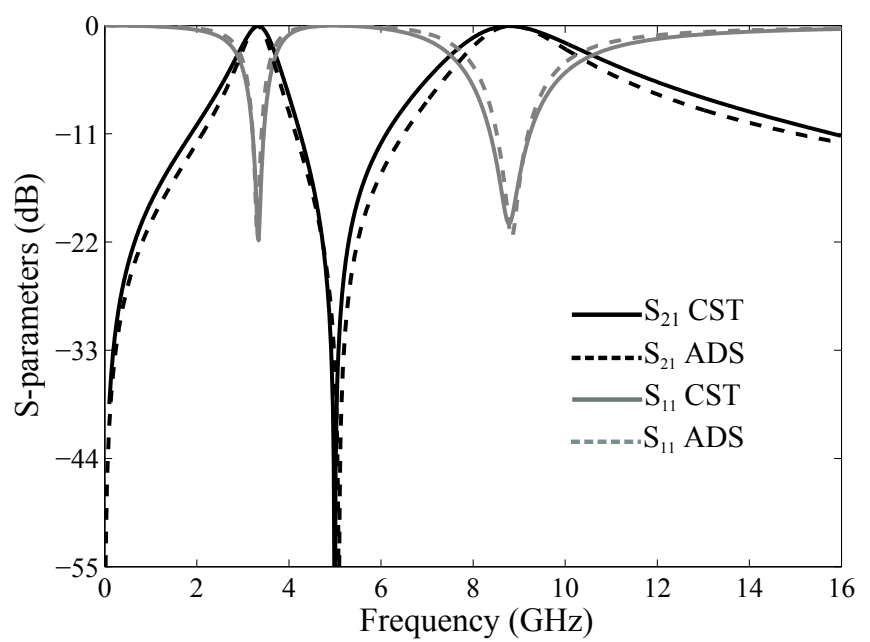

Fig. 3. Free space full wave simulation result of the designed dual-band FSS using periodic boundary condition in CST in comparison with the ADS circuit model simulation results.

by a distance of $g$. The gap between the unit cells show a capacitive effect to the incident wave. This capacitive effect is denoted by $C_{1}$ of the hybrid resonator where, increasing $g$ causes a smaller $C_{1}$. In addition, the loop and square patch in each unit cell provide the parallel $L_{1} C_{1}$ resonance behaviour where $L_{1}$ is provided by the inductive effect of the loop sides and $C_{1}$ is provided by the capacitive effect between the square patch and the loop sides. In the top pattern, increasing $w_{r}$ causes a smaller $L_{2}$ and decreasing $D_{P}$ causes a smaller $C_{2}$.

TABLE I

Physical and Equivalent Circuit Parameters of the Designed DUAL-BAND FSS

\begin{tabular}{cccccc}
\hline Parameter & $g$ & $w$ & $w_{r}$ & $D_{x}, D_{y}$ & $D_{P}$ \\
\hline Value & $0.1 \mathrm{~mm}$ & $0.2 \mathrm{~mm}$ & $0.15 \mathrm{~mm}$ & $8.8 \mathrm{~mm}$ & $8 \mathrm{~mm}$ \\
\hline Parameter & $L_{1}$ & $L_{2}$ & $C_{1}$ & $C_{2}$ & $Z_{T}$ \\
\hline Value & $2.8 \mathrm{nH}$ & $0.98 \mathrm{nH}$ & $0.54 \mathrm{pF}$ & $0.46 \mathrm{pF}$ & $254 \Omega$ \\
\hline
\end{tabular}




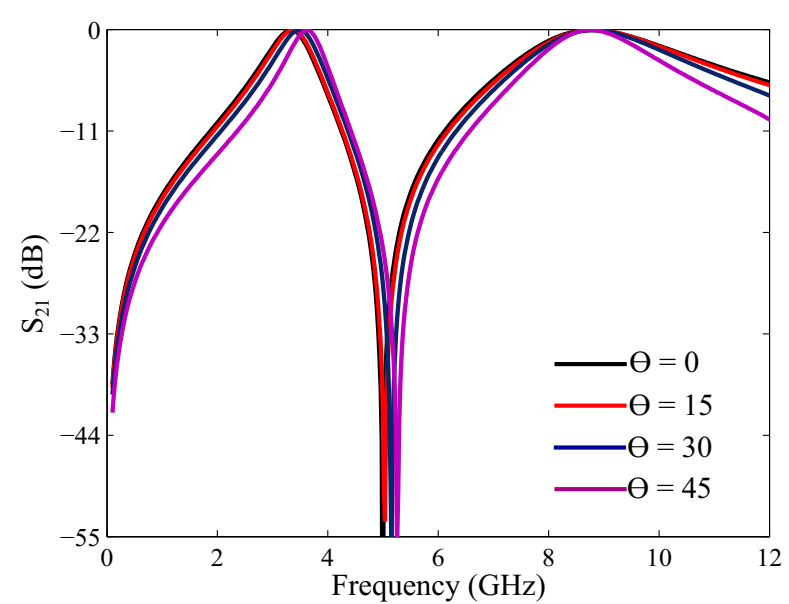

Fig. 4. Full wave simulation results of the transmission coeficient of the designed FSS as function of the electromagnetic wave incident angle.

\section{Simulation Results And Discussions}

Based on the circuit model and the procedure discussed above, a dual-band FSS with passbands at S- and X-bands with central frequencies of 3.3 and $9.5 \mathrm{GHz}$ has been designed and simulated in CST Microwave Studio full wave simulator. The physical parameters of the designed FSS are listed in Table I together with its equivalent circuit parameters values. The top and bottom metallic layers are patterned on the both sides of $0.254 \mathrm{~mm}$ thick Rogers RO5880 dielectric substrate with the relative permittivity of $\epsilon_{r}=2.2$ and loss tangent of $\tan \delta=0.0009$. The full wave simulations are carried out using unit cell boundary condition that simulates an infinite array of unit FSS cells that are exposed to an arbitrary incident angle electromagnetic wave. On the other hand, the equivalent circuit of the FSS (Fig. 1) is simulated in ADS using the parameters listed in Table I. These circuit parameters are optimized by curve fitting using ADS. The full wave simulation results of the FSS are plotted in Fig. 3 together with the results obtained by using ADS. As seen, there is a good agreement between the full wave and circuit model simulations. The passband frequencies of the filter are 3.3 and $8.6 \mathrm{GHz}$ with transmission zero at around $5 \mathrm{GHz}$ that improves the out of band rejection of the FSS.

The scan performance of the designed FSS is shown in Fig. 4 showing a low sensitivity to oblique angle of the incident electromagnetic wave. This low sensitivity arises from the miniaturized unit cell size since the unit cell dimension is as small as $\lambda_{0} / 10$ where, $\lambda_{0}$ is the wavelength at the first operational passband. This feature provides a freedom to use the designed FSS in the applications with non-planar incident phase fronts [13].

As discussed in Section II, the two passband frequencies of the proposed FSS can be controlled independently by controlling $C_{1}$ and $C_{2}$ element values. The value of the $C_{1}$ capacitor can be tuned by varying the gap between adjacent square-loop unit cells $(g)$ where decreasing $g$ increases $C_{1}$ and shifts the first passband frequency downward without affecting $f_{2}$. This phenomenon is investigated using the full simulations in CST by changing $g$ and the results are shown in Fig. 5. As

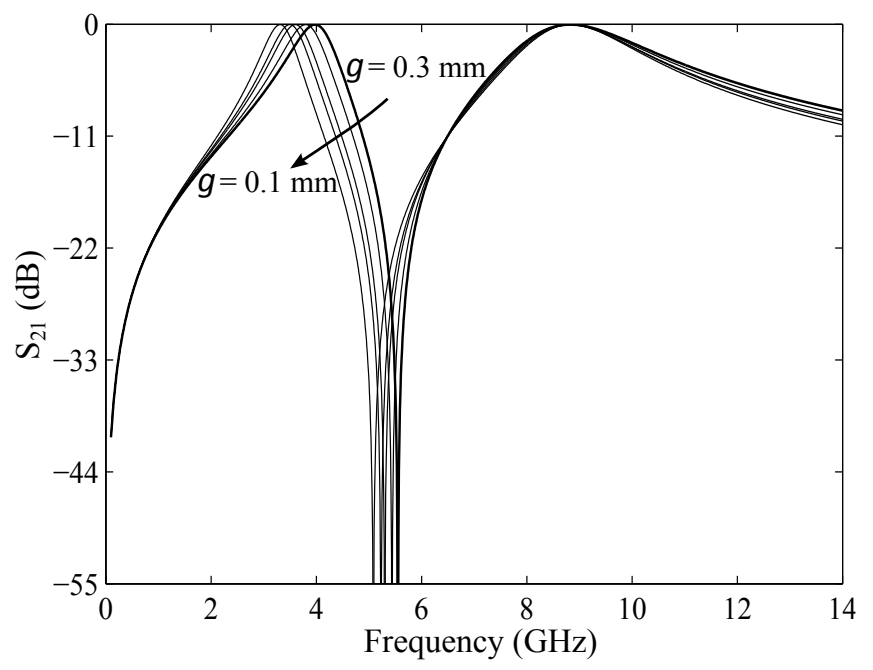

Fig. 5. Full wave simulation of the transmission coeficient of the dual-band FSS as a function of the $g$. In simulations $g$ is varied from $0.1 \mathrm{~mm}$ to $0.3 \mathrm{~mm}$ with a step size of $0.05 \mathrm{~mm}$.

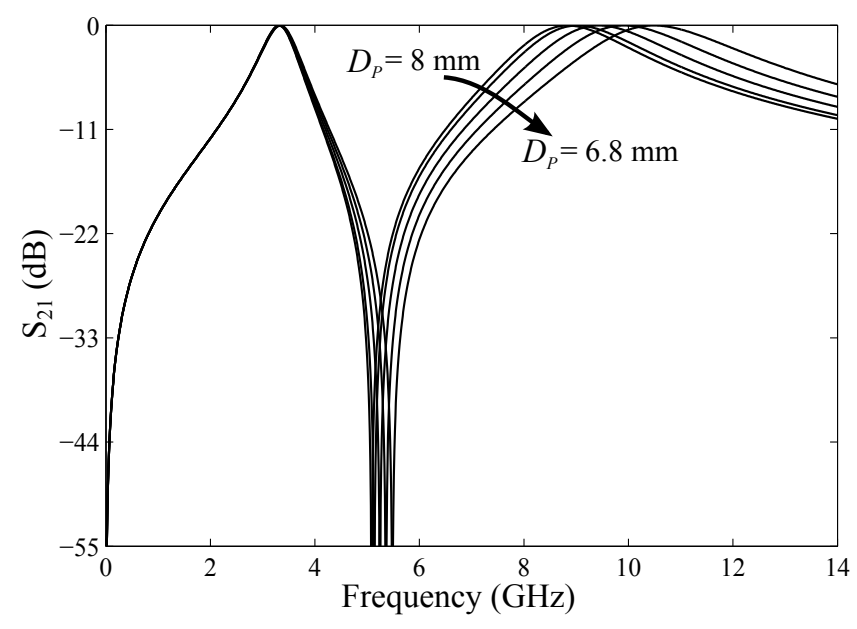

Fig. 6. Full wave simulation of the transmission coeficient of the dual-band FSS as a function of the $D_{P}$. In simulations $D_{P}$ is varied from $8 \mathrm{~mm}$ to $6.8 \mathrm{~mm}$ with a step size of $0.2 \mathrm{~mm}$.

demonstrated in Fig. 5, the larger value of $g$ causes a lower $f_{1}$ without a significant effect on the second passband frequency.

On the other hand, the $C_{2}$ capacitor can be tuned by changing the square patch size $D_{P}$. A larger $D_{P}$ causes a larger $C_{2}$ which shifts the upper passband frequency downward without sensible change in $f_{1}$. Fig. 6 demostrates the full wave simulation results of $D_{P}$ variations effect on the FSS transmission response. As seen, a larger $D_{P}$ leads to a lower $f_{2}$ without significant variation in the lower passband frequency, confirming the analysis presented in Section II.

\section{CONClusion}

A new concept of miniaturized element dual-band frequency selective surface has been presented and analysed by considering its accurate equivalent circuit model. The designed FSS incorporates the unit cells with dimensions as small as $\lambda_{0} / 10$ at the first operational passband providing desired independency of the transmission response with respect to the 
angle of the incident wave. In addition, the proposed design approach gives the benefit of independent controllability of the two passband frequencies simply by tuning the unit cell physical parameters.

\section{REFERENCES}

[1] F. Bayatpur and K. Sarabandi, "Single-layer high-order miniaturizedelement frequency-selective surfaces," IEEE Transactions on Microwave Theory and Techniques, vol. 56, no. 4, pp. 774-781, 2008.

[2] A. Ebrahimi, P. Yaghmaee, W. Withayachumnankul, C. Fumeaux, S. AlSarawi, and D. Abbott, "Interlayer tuning of X-band frequency-selective surface using liquid crystal," in Proceedings of IEEE 2013 Asia-Pacific Microwave Conference (APMC2013), 2013.

[3] B. Li and Z. Shen, "Synthesis of quasi-elliptic bandpass frequencyselective surface using cascaded loop arrays," IEEE Transactions on Antennas and Propagation, vol. 61, no. 6, pp. 3053-3059, 2013.

[4] M. Li and N. Behdad, "A third-order bandpass frequency selective surface with a tunable transmission null," IEEE Transactions on Antennas and Propagation, vol. 60, no. 4, pp. 2109-2113, 2012.

[5] Y. Zuo, A. K. Rashid, Z. Shen, and Y. Feng, "Design of dual-polarized frequency selective structure with quasi-elliptic bandpass response," IEEE Antennas and Wireless Propagation Letters, vol. 11, pp. 297-300, 2012.

[6] P. Yaghmaee, W. Withayachumnankul, A. K. Horestani, A. Ebrahimi, B. Bates, and C. Fumeaux, "Tunable electric-lc resonators using liquid crystal," Proceedings of the IEEE International Symposium on Antenna and Propagation (APS/USNC-URSI13), 2013.

[7] M. Al-Joumayly and N. Behdad, "Low-profile, highly-selective, dualband frequency selective surfaces with closely spaced bands of operation," IEEE Transactions on Antennas and Propagation, vol. 58, no. 12, pp. 4042-4050, 2010.

[8] J. Gianvittorio, Y. Rahmat-Samii, and J. Romeu, "Fractal FSS: various self-similar geometries used for dual-band and dual-polarized FSS," in Proceedings of IEEE Antennas and Propagation Society International Symposium, 2001, vol. 3, 2001, pp. 640-643 vol.3.

[9] J. Romeu and Y. Rahmat-Samii, "Fractal FSS: a novel dual-band frequency selective surface," IEEE Transactions on Antennas and Propagation, vol. 48, no. 7, pp. 1097-1105, 2000.

[10] R. Hill and B. Munk, "The effect of perturbating a frequency-selective surface and its relation to the design of a dual-band surface," IEEE Transactions on Antennas and Propagation, vol. 44, no. 3, pp. 368 374, 1996.

[11] G. Manara, A. Monorchio, and R. Mittra, "Frequency selective surface design based on genetic algorithm," Electronics Letters, vol. 35, no. 17, pp. 1400-1401, 1999.

[12] J. A. Bossard, D. H. Werner, T. S. Mayer, and R. P. Drupp, "A novel design methodology for reconfigurable frequency selective surfaces using genetic algorithms," IEEE Transactions on Antennas and Propagation, vol. 53, no. 4, pp. 1390-1400, 2005.

[13] F. Bayatpur and K. Sarabandi, "Multipole spatial filters using metamaterial-based miniaturized-element frequency-selective surfaces," IEEE Transactions on Microwave Theory and Techniques, vol. 56, no. 12 , pp. $2742-2747,2008$.

[14] M. Salehi and N. Behdad, "A second-order dual x-/ka-band frequency selective surface," IEEE Microwave and Wireless Components Letters, vol. 18, no. 12, pp. 785-787, 2008. 